Supplementary Information for

\title{
Rotational Locomotion of an Active Gel Driven by Internal
}

\section{Chemical Signals}

Jing Wang, ${ }^{a, \#}$ Lin Ren, ${ }^{b, \#}$ Rui Teng, ${ }^{a}$ Irving R Epstein,,${ }^{c}{ }^{*}$ Hui Wang, ${ }^{a}$ Meng Zhang, ${ }^{a}$ Ling Yuan, ${ }^{a}$ Qingyu Gao ${ }^{a}{ }^{*}$

a College of Chemical Engineering, China University of Mining and Technology, Xuzhou, 221116 Jiangsu, China

${ }^{b}$ College of Chemistry and Materials Engineering, Wenzhou University, Wenzhou, 325035 Zhejiang, China

c Department of Chemistry and Volen Center for Complex Systems, Brandeis University, Waltham, 02454-9110 Massachusetts, USA

[\#] These authors contributed equally to this work.

*Email: epstein@brandeis.edu, gaoqy@cumt.edu.cn.

\section{Table of Contents}

Materials and synthesis for nano-based PAAm self-oscillating BZ gels........................S2

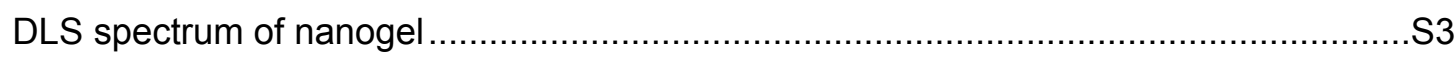

Image collection and locus tracking for gel locomotion .................................................... 3

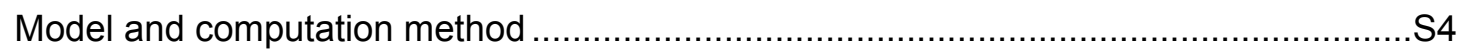

Phase difference between time series of the horizontal $(x)$ and vertical $(y)$ displacements

Detailed explanation and calculation of phase difference of perpendicular locomotion components in 2D BZ gel ..S6

Supplementary movies S8

References ..S8 


\section{Materials and Synthesis for nano-based PAAm self-oscillating BZ Gels}

1.1 Materials Acrylamide (AAm) (Shanghai Aladdin Biochemical Technology Co. Ltd.), N,N'methylenebis (acrylamide) (MBAA), sodium dodecyl sulfonate (SDS), azodiisobutyronitrile (AIBN), dimethyl sulfoxide (DMSO), malonic acid (MA), sodium bromate $\left(\mathrm{NaBrO}_{3}\right)$, and nitric acid $\left(\mathrm{HNO}_{3}\right)$ were all purchased from Sinopharm Chemical Reagent Co., Ltd. and used without further purification. Ruthenium (4-vinyl-4'-menthyl-2,2'-bipyridine) bis (2,2'bipyridine) bis (hexafluorophosphate) $\left(\mathrm{Ru}(\mathrm{bpy})_{3}{ }^{2+}\right.$ ) was synthesized according to the literature, ${ }^{1}$ Milli-Q water $(18.2 \mathrm{M} \Omega)$ was used in all experiments.

1.2 Synthesis of PAAm-activated nanogels. PAAm activated nanogels were synthesized according to the method of reference ${ }^{2}$ by changing the monomer to AAm and the initiator to AIBN. A schematic for the synthesis of the PAAm self-oscillating BZ gel in this study is shown in Scheme S1. ${ }^{2}$ First, AAm and MBAA were added to $50 \mathrm{~mL}$ of deaerated water $([\mathrm{AAm}]=0.2 \mathrm{mmol} / \mathrm{mL}$ and $[\mathrm{MBAA}]=0.008 \mathrm{mmol} / \mathrm{mL})$. After stirring and dissolving the above mixture, $1 \mathrm{~mL}$ methanol solution containing $0.1 \mathrm{mmol}$ AIBN was added to the solution, and then the surfactant SDS (1 mmol) was added. When the solution was uniform, the water bath was heated to $60^{\circ} \mathrm{C}$, and the activated nanogel with unsaturated bonds was obtained after $35 \mathrm{~min}$. The activated nanogel was stored at $0{ }^{\circ} \mathrm{C}$.

1.3 Synthesis of nano-based PAAm self-oscillating BZ gels. In the first step, AAm (2 mmol) and $\mathrm{Ru}(\mathrm{bpy}) \mathrm{3}^{2+}(0.013 \mathrm{mmol})$ were dissolved in DMSO. After the solid was dissolved completely by ultrasound, the activated nanogel solution was slowly added into the mixed solution. In the second step, the mixed solution was rapidly injected into a glass tube with a diameter of $1.0 \mathrm{~mm}$, and the ends of the tube were sealed and then polymerized at $60{ }^{\circ} \mathrm{C}$ for $18 \mathrm{~h}$. After gelation, the resulting PAAm self-oscillating gel was soaked in methanol to remove unreacted compounds and then stored in pure water. 


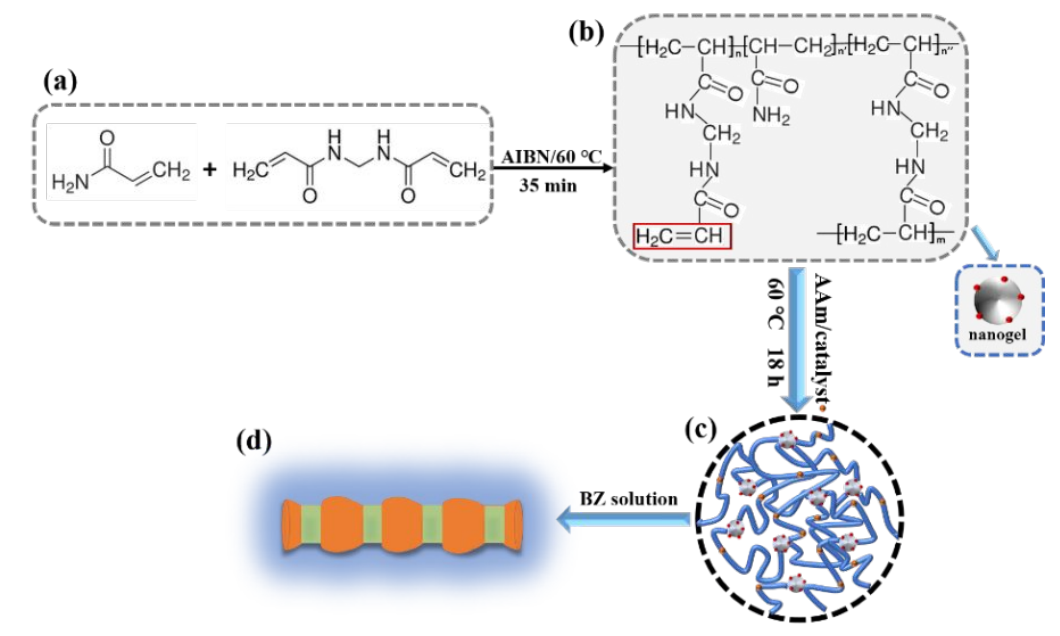

Figure S1. Schematic diagram of nano-based PAAm self-oscillating BZ gel synthesis. (a) Main reagents for activated nanogel synthesis. (b) Chemical structure of the activated nanogel with unsaturated double bonds from the unreacted parts of MBAA. (c) Schematic diagram of gel spatial structure. (d) In the $\mathrm{BZ}$ solution, the gel swells in $\mathrm{Ru}^{2+}$ (orange) and deswells in $\mathrm{Ru}^{3+}$ (green).

\section{DLS spectrum of nanogel}

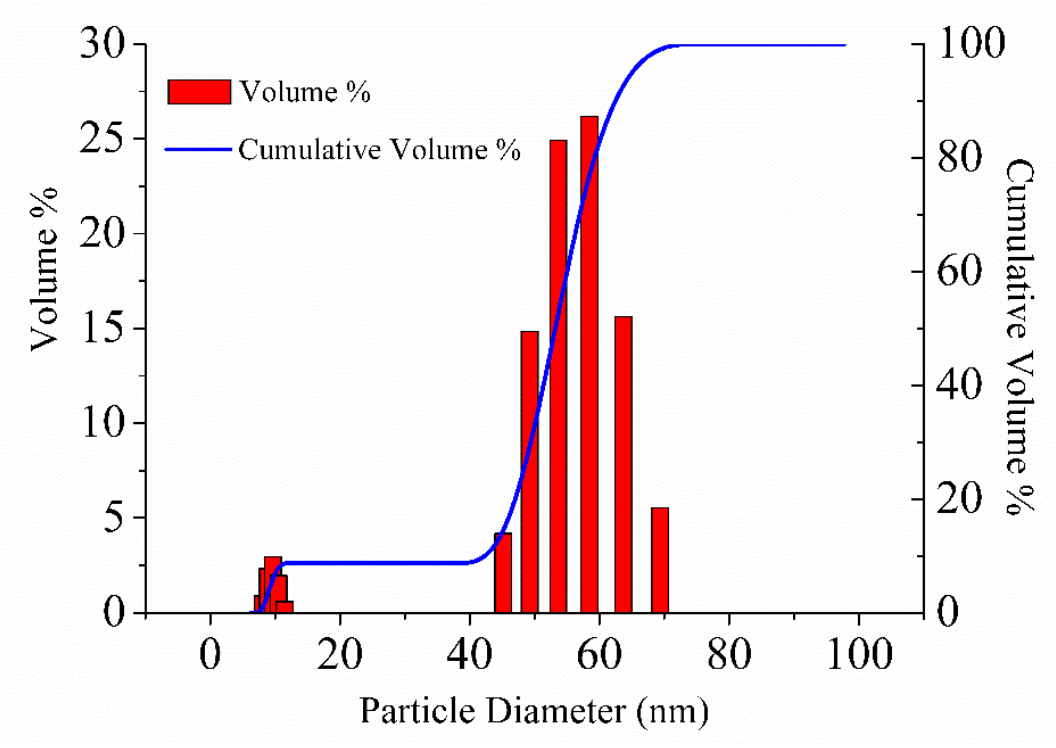

Figure S2. DLS spectrum of the nanogel at a reaction time of $35 \mathrm{~min}$; particle size distribution is $45.0-70.0 \mathrm{~nm}$ with an average particle size of $53.4 \mathrm{~nm}$.

\section{Image collection and locus tracking for gel locomotion}

The diameter of the quartz reactor is $6 \mathrm{~cm}$. The purified gel is placed in $30 \mathrm{~mL} \mathrm{BZ}$ solution containing $\mathrm{MA}, \mathrm{HNO}_{3}$ and $\mathrm{NaBrO}_{3}$ (without catalyst), and the circulating water temperature is 22.0 $\pm 0.1{ }^{\circ} \mathrm{C}$. The light environment is provided by a white LED coaxial light source, and the intensity of the light source is controlled by a digital control unit (LOTS). The image is recorded with an 
industrial camera (Mind Vision) connected to the computer. An image with position data of the gel center is collected every 3s. Image data are processed with MATLAB software after importing the photos into MATLAB. After processing with the function rgb2gray and binarization with $i m 2 b w$, we obtain the binary image matrix. The experimental video is made from the collected images with a play speed of 6 frames per second. The built-in function regionprops is used to track the center point of the gel in the image. By superposing the centroids of the gel in each image, the movement track of the gel is obtained.

\section{Model and computation method}

Our PAAm-based BZ gel model is constructed by coupling the Yashin-Balazs model (gLSM) ${ }^{3-}$ ${ }^{5}$ with the two-variable form of the Amemiya model for the photosensitive BZ reaction. ${ }^{6,7}$ The contraction of the PAAm gel is produced from the formation of additional reversible crosslinks generated by the oxidation of the Ru (II) catalyst. To simplify the model, the effects of the total concentration of the oxidized catalyst on the gel are not considered here. The governing partial differential equations (PDEs) for 2D gels are expressed as follows:

$$
\left\{\begin{array}{l}
\mathrm{d} \phi / \mathrm{d} t=-\phi \nabla \overrightarrow{\boldsymbol{V}^{\mathrm{p}}} \\
\mathrm{d} u / \mathrm{d} t=-u \nabla \overrightarrow{\boldsymbol{V}^{\mathrm{p}}}+\nabla\left(u \overrightarrow{\boldsymbol{V}^{\mathrm{p}}} /(1-\phi)\right)+\nabla((1-\phi) \nabla(u /(1-\phi)))+F(u, v, \phi, I) \\
\mathrm{d} v / \mathrm{d} t=-\nu \nabla \overrightarrow{\boldsymbol{V}^{\mathrm{p}}}+G(u, v, \phi, I)
\end{array}\right.
$$

$\hat{\boldsymbol{\sigma}}=-P(\phi, v) \hat{\mathbf{I}^{+}} c_{0}(v) v_{0} \phi_{0}^{-1} \hat{\phi_{\mathrm{B}}}$

$P(\phi, v)=\pi_{\mathrm{osm}}(\phi)+c_{0}(v) v_{0} \phi\left(2 \phi_{0}\right)^{-1}$

$\pi_{\mathrm{osm}}(\phi)=-\left[\phi+\ln (1-\phi)+\chi_{0} \phi^{2}\right]$

$\overrightarrow{\boldsymbol{V}^{\mathrm{p}}}=\nabla \hat{\boldsymbol{\sigma}} / \zeta^{\prime}(\phi)$

$\zeta^{\prime}(\phi)=(\Lambda 0(1-\phi))^{-1}\left(\phi / \phi_{0}\right)^{3 / 2}$

$c_{0}(v)=(c$ bis $0+b v)$

where ${ }^{3-7} u$ and $v$ are the nondimensionalized concentrations of $\mathrm{HBrO}_{2}$ and $\mathrm{Ru}(\mathrm{bpy})_{3}{ }^{3+}$ in the Oregonator model, respectively. $\phi$ is the volume fraction of the gel. $\hat{\boldsymbol{\sigma}}, \hat{\mathrm{B}}, \hat{\mathrm{I}}$ and $\nabla \hat{\boldsymbol{\sigma}}$ denote the stress tensor, strain tensor, unit tensor and stress gradient, respectively. $\sigma 2 \mathrm{D}$ el is the elastic force, $c_{0}$ is the dynamic cross-link density, and $c_{0}{ }^{\text {bisis }}$ the density of the permanent crosslinks. ${ }^{8}$ In our model, the density of $\mathrm{Ru}(\mathrm{III})$-induced additional reversible crosslinks is proportional to the $v$ variable. ${ }^{8} v_{0}$ denotes the volume of a monomeric unit within the polymer chains, which is normalized in the calculation. $\overrightarrow{V^{\mathrm{p}}}$ is the velocity vector of the polymer. The apparent friction coefficient, $\zeta^{\prime}(\phi)$, is a function of $\phi, \Lambda_{0}$ is a dimensionless kinetic coefficient set to 100 , and $\phi_{0}$ denotes the undeformed 
polymer volume fraction. The functions $\mathrm{F}$ and $\mathrm{G}$ describe the reaction kinetics of the $\mathrm{BZ}$ reaction (modified Oregonator model). We replace the simple Oregonator in the Yashin-Balazs model with a two-variable photosensitive Oregonator model that incorporates both photoinduction and photoinhibition in the $\mathrm{Ru}$ (bipy)-catalyzed BZ reaction. The functions $F(u, v, \phi, I)$ and $G(u, v, \phi, I)$ used to describe the gel-coupled $\mathrm{BZ}$ reactions are expressed as follows:

$F(u, v, \phi, I)=(1-\phi)^{2} u-u^{2}-\left(f v+I P_{1}\right)(1-\phi)\left[u-q(1-\phi)^{2}\right]\left[u+q(1-\phi)^{2}\right]^{-1}+I P_{2}$

$G(u, v, \phi, I)=\varepsilon\left[(1-\phi)^{2} u-(1-\phi) v+I\left(0.5 P_{1}+P_{2}\right)\right]$

Here, $\varepsilon, f$ and $q$ are the Oregonator parameters. $I$ denote the dimensionless homogeneous light intensity. $P_{1}$ and $P_{2}$ are the rate coefficients that characterize the two key photochemical reaction steps.

The diffusion coefficient $D=1.5 \times 10^{-5} \mathrm{~cm}^{2} / \mathrm{s},{ }^{9} T_{0}=1.0 /\left(k_{3}\left[\mathrm{H}^{+}\right]\left[\mathrm{BrO}_{3}^{-}\right]\right)$is set at $0.6 \mathrm{~s}$, and $L_{0}=$ $\left(D T_{0}\right)^{0.5} 0.03 \mathrm{~mm}$. The gel size is $L_{\mathrm{x}} \times L_{\mathrm{y}}=5.0 \mathrm{~mm} \times 1.8 \mathrm{~mm}$. Note that the spatiotemporal data shown in both the simulated plots and the movie are dimensional values, while the $v$ value is dimensionless. Other default values used for our model simulation are $f=1.0, \varepsilon=0.13, P_{1}=0.124, P_{2}=0.77, \boldsymbol{c}_{0}^{\text {bis }}$ $=1.0 \times 10^{-4}, b=0.075$, and $\lambda_{\perp}=1.1$.

For the setting of the gel boundary conditions, we refer to reference 9 and apply zero-flux boundary conditions. To obtain stable spiral waves consistent with the experiments, we generate spiral waves by the cross method along the edges of the gel (initially setting a gradient of $u$ along the $x$-direction and a gradient of $v$ along the $y$ axis of the gel). The homogeneous light intensity $I=1.0 \times 10^{-5}$.

5. Phase difference between time series of the horizontal ( $x$ ) and vertical (y) displacements (a)

(b)

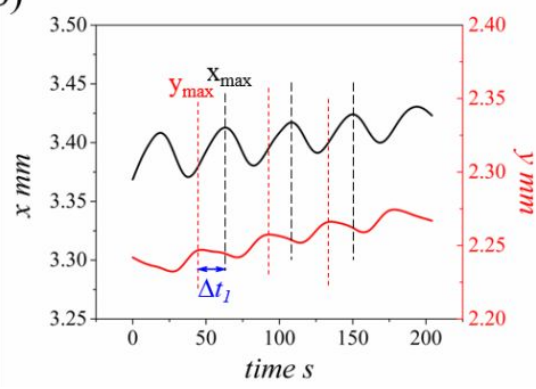

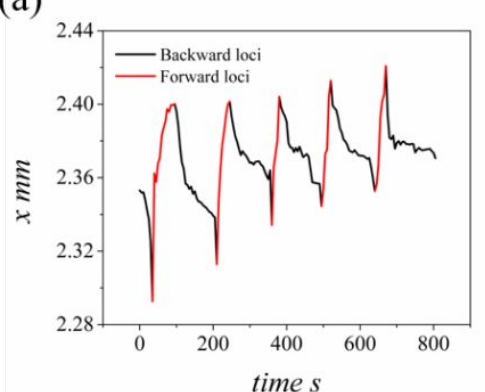

(c)

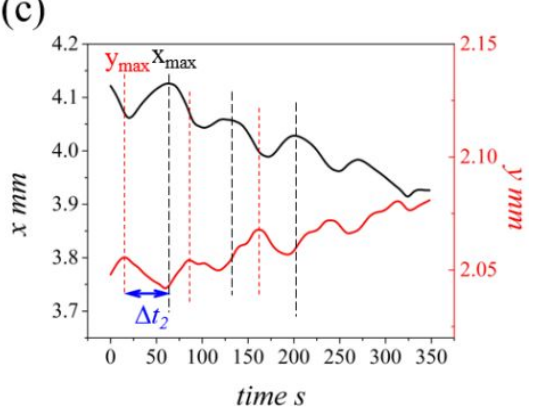


Figure S3. Experimental time series for $\mathrm{x}$ and $\mathrm{y}$ displacements in 1D and 2D spaces. (a) 1D gel locomotion; Experimental conditions: $[\mathrm{MA}]_{0}=0.1 \mathrm{M},\left[\mathrm{HNO}_{3}\right]_{0}=0.7 \mathrm{M}$, and $\left[\mathrm{NaBrO}_{3}\right]_{0}=0.2 \mathrm{M}$. The temperature is $22 \pm 0.1^{\circ} \mathrm{C}$. (b and c) $2 \mathrm{D}$ locomotion driven by spiral waves and pulse waves $(\mathrm{b}$ for Figure 1a, c for Figure 2a), respectively.

\section{Detailed explanation and calculation of phase difference of perpendicular locomotion components in $2 \mathrm{D} \mathrm{BZ}$ gel}

During locomotion of a two-dimensional spatial BZ gel driven by traveling waves (Figure S4a), local oscillations at every point have the same oscillatory period. Phase asynchrony of different local dynamics for gel stimulus $(v)$ is caused by the traveling wave structure as shown in Figure S4b (points $\mathrm{i}, \mathrm{j}$ and $\mathrm{k}$ are marked in Figure S4a), so the difference of stimulus concentration between nearby spatial points is also oscillatory in time, and phase differences occur between oscillations of $v_{i}-v_{j}, v_{j}-v_{k}$ and $v_{k}-v_{i}$ as shown in Figure S4c. Similarly, phase differences are found between local oscillations of spatial derivatives $\left(v_{x}\right.$ and $\left.v_{y}\right)$ in the two perpendicular directions at any point (Figure S5a-c).

(a)

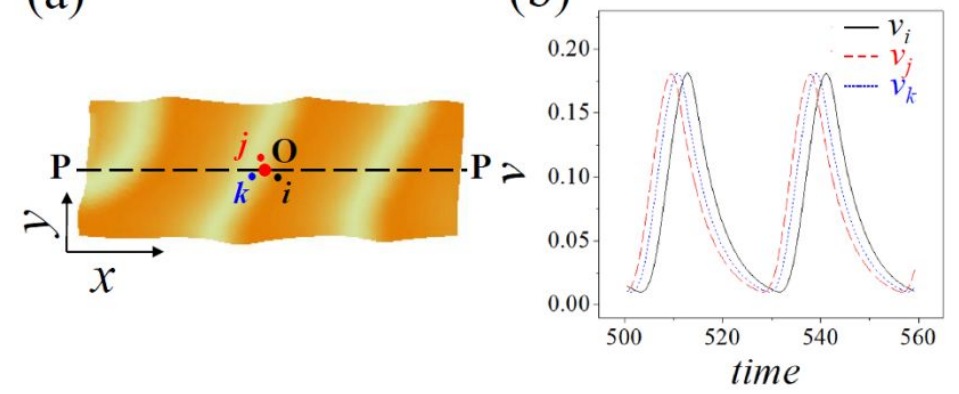

(c)

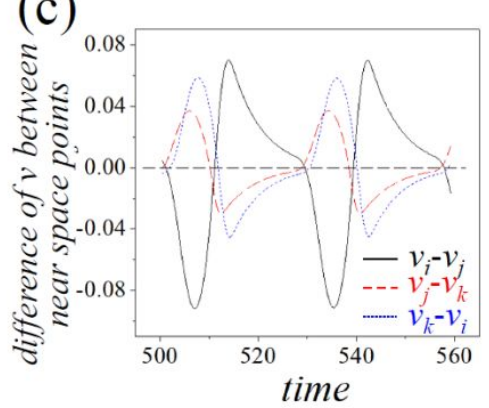

Figure S4. Traveling waves and simulated time-series of oscillations at several points in a BZ gel. (a) Traveling waves in rectangular BZ gel. (b) Time series of $v$-oscillations at points $i, j$ and $k\left(v_{i}, v_{j}\right.$ and $v_{k}$, respectively). (c) Time series of the difference of $v$ between nearby spatial points, i.e., $v_{i}-v_{j}$, $v_{j}-v_{k}$ and $v_{k}-v_{i}$.

According to the gLSM of the BZ gel, ${ }^{3-5,8}$ the dynamic cross-link density $c_{0}$ in $\nabla \hat{\boldsymbol{\sigma}}$ has a dynamical term $b \times v^{\prime}$, where $v^{\prime}$ is the spatial derivative of the gel stimulus, which is the origin of the driving force. There is an approximate linear relationship between $v$ ' and the driving force $(F) .{ }^{4}$ The velocity of the gel, $\overrightarrow{V^{\mathrm{p}}}$, is dominated by $\nabla \hat{\boldsymbol{\sigma}}$ as shown in Equation S1. Note that the $2 \mathrm{D}$ form of this relationship in gLSM is: ${ }^{4}$

$$
\boldsymbol{V e l}=M F
$$

Where $\mathbf{V e l}$ is the velocity of gel's grid, the mobility $M$ is a function of $\phi$ (M changes only slightly during the gel locomotion), so the grid velocity $\mathbf{V e l}$ is proportional to the force $F$. The above relationships among the spatial derivative $(v ')$ of the stimulus concentration, the local driving force 
and the gel velocity imply that the last two quantities should also show phase differences between the oscillations of their corresponding components in the $x$ and $y$ directions. The calculated results are shown in Figure S5d-i.

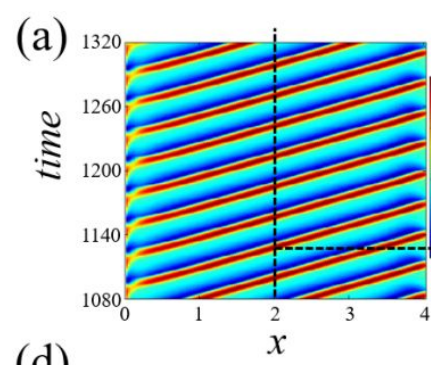

(d)

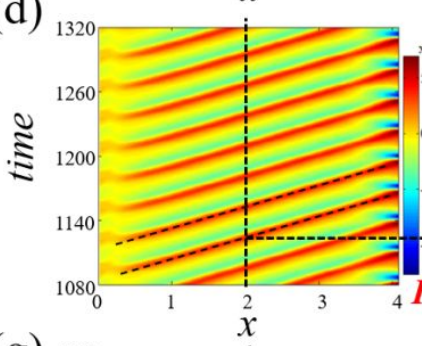

(b)

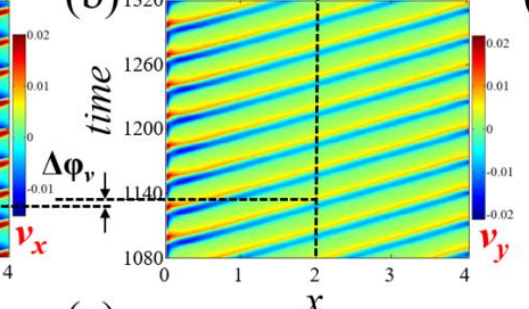

(e)

(g)

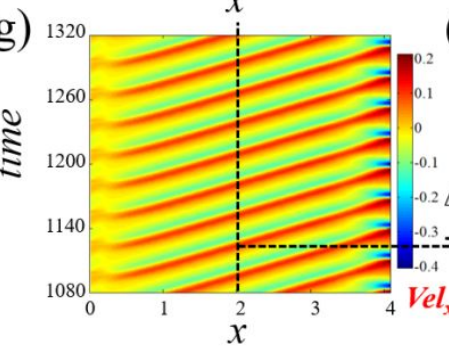

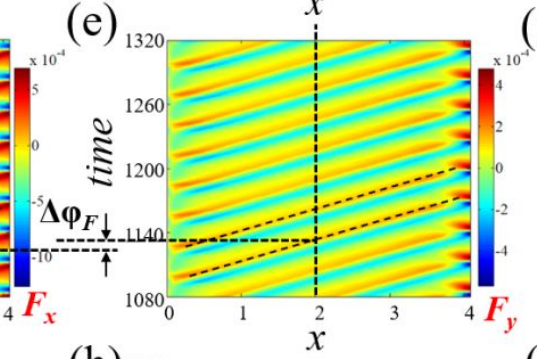

(h) 13

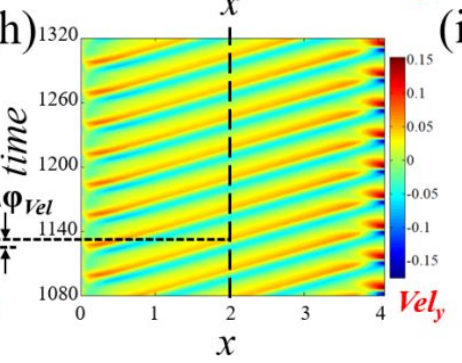

(c)

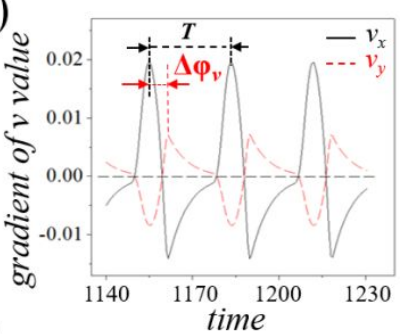

(f)

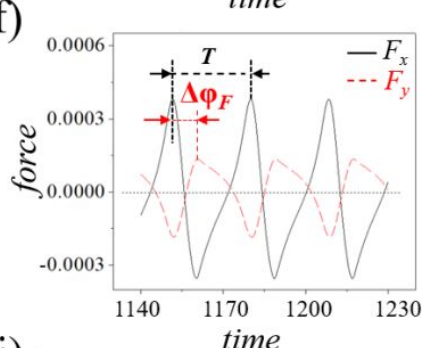

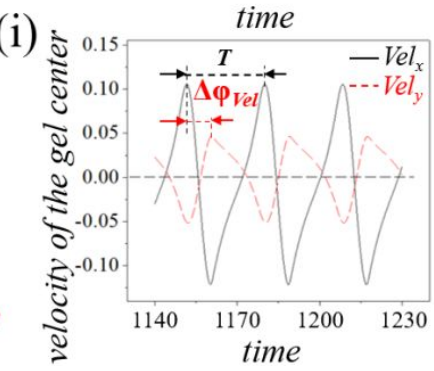

Figure S5. Spatiotemporal plots and local time series of the spatial gradient of $v$, force and velocity in the gel. Each space-time plot consists of 150 horizontal data scans along the P-P line in Figure S4a at $0.6 \mathrm{~s}$ intervals. Spatiotemporal plots of (a) $v_{\mathrm{x}}$, (b) $v_{\mathrm{y}}$, (d) $F_{\mathrm{x}}$, (e) $F_{\mathrm{y}}$, (g) $\boldsymbol{V e l}_{\mathrm{x}}$, (h) Vel $\boldsymbol{V}_{\mathrm{y}}$. Time series of (c) $v_{\mathrm{x}}$ and $v_{\mathrm{y}}$, (f) $F_{\mathrm{x}}$ and $F_{\mathrm{y}}$, (i) $\boldsymbol{V e} \boldsymbol{e}_{\mathrm{x}}$ and $\boldsymbol{V e} \boldsymbol{e}_{\mathrm{y}}$, at position $x=2.0 \mathrm{~mm}$ from the left boundary (see Figure S4a).

Ultimately, then, because of the linear relation between velocity and displacement, oscillations of peristaltic motion in the $x$ and $y$ directions should behave in the same way, as seen in Figure S6. 


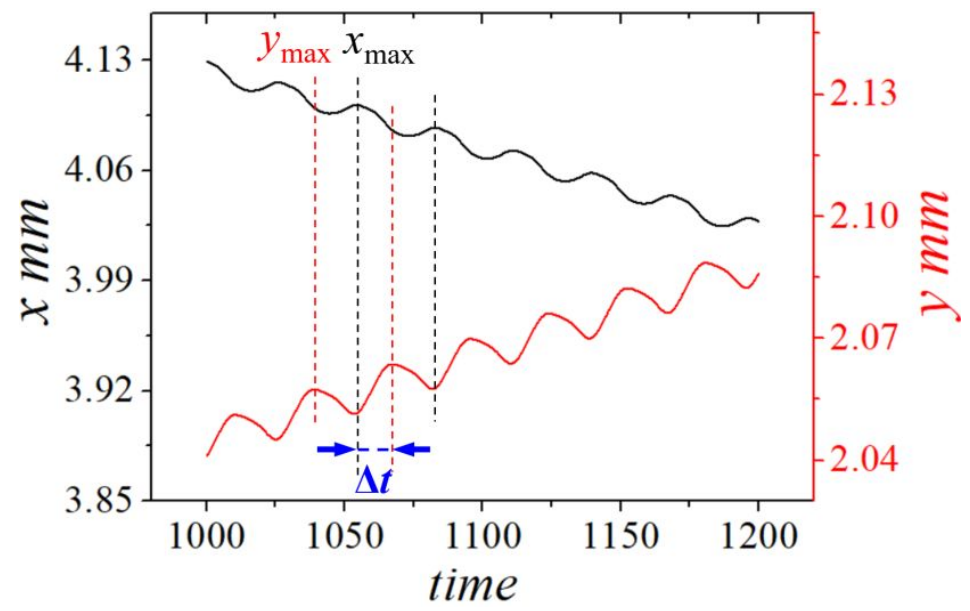

Figure S6. Time series for $x$ and $y$ displacement in 2D simulated gel locomotion.

In summary, phase differences of peristaltic displacement between the perpendicular components $(x$ and $y$ ) produce active rotational locomotion driven by traveling waves, as the result of phase asynchrony of spatial oscillatory dynamics in two- or three-dimensional space. We note additionally that if pulse waves were initiated at the midpoint of the $y$ boundary, the symmetry in the spatial derivative $\left(v_{\mathrm{y}}\right)$ would lead to zero displacements along the $y$-direction, i.e., onedimensional locomotion along the $x$-direction. However, spiral waves driven gel locomotion always show rotational behavior with any initiation point on account of the spatial asymmetry of the spiral dynamics.

\section{Supplementary movies}

Movie S1. Experimental rotational locomotion of a PAAm BZ gel driven by spiral waves.

Movie S2. Numerical rotational locomotion of a PAAm BZ gel driven by spiral waves.

Movie S3. Experimental rotational locomotion of a PAAm BZ gel driven by pulse waves.

Movie S4. Numerical rotational locomotion of a PAAm BZ gel driven by pulse waves.

\section{References}

1. Shinohara, S.; Seki, T.; Sakai, T.; Yoshida, R.; Takeoka, Y. Photo regulated wormlike motion of a gel. Angew. Chem., Int. Ed. 2008, 120, $9179-9183$.

2. Xia, L.; Xie, R.; Ju, X.; Wang, W.; Chen, Q.; Chu, L. Nano-structured smart hydrogels with rapid response and high elasticity. Nat. Commun. 2013, 4, 375-381.

3. Yashin, V. V.; Balazs, A. C. Modeling Polymer Gels Exhibiting Self-Oscillations Due to the Belousov-Zhabotinsky Reaction. Macromolecules 2006, 39, 2024-2026.

4. Yashin, V. V.; Balazs, A. C. Theoretical and computational modeling of self-oscillating 
polymer gels. J. Chem. Phys. 2007, 126, 1-144.

5. Kuksenok, O.; Yashin, V. V.; Balazs, A. C. Three-dimensional model for chemoresponsive polymer gels undergoing the Belousov-Zhabotinsky reaction. Phys. Rev. E. 2008, 78, 041406.

6. Lu, X.; Ren, L.; Gao, Q.; Zhao, Y.; Wang, S.; Yang, J.; Epstein, I. R. Photophobic and phototropic movement of a self-oscillating gel. Chem. Commun. 2013, 49, 7690-7692.

7. Amemiya, T.; Ohmori, T.; Nakaiwa, M.; Yamaguchi, T. J. Phys. Chem. A 1998, 102, 10 45374542

8. Yuan, P.; Kuksenok, O.; Gross, D. E.; Balazs, A. C.; Moore, J. S.; Nuzzo, R. G. UV patternable thin film chemistry for shape and functionally versatile self-oscillating gels Soft Matter 2013, 9, 1231-1243.

9. Yoshida, R.; Takahashi, T.; Yamaguchi, T.; Ichijo, H. Self-Oscillating Gel. J. Am. Chem. Soc. 1996, 118, 5134-5135. 